\title{
Selected articles from the IEEE International Workshop on Genomic Signal Processing and Statistics (GENSIPS'2011)
}

\author{
Ranadip Pal ${ }^{1 *}$, Yufei Huang ${ }^{2}$, Yidong Chen $^{3}$ \\ From IEEE International Workshop on Genomic Signal Processing and Statistics (GENSIPS) 2011 \\ San Antonio, TX, USA. 4-6 December 2011
}

\begin{abstract}
Introduction
The 2011 IEEE International Workshop on Genomic Signal Processing and Statistics (GENSIPS 2011) was organized in San Antonio, Texas from December 2-4th. GENSIPS'11 provided a forum for researchers in the signal processing community, other related computational experts, and biomedical scientists to exchange ideas and discuss signal processing challenges due to the high modality of disparate high-throughput data, high variability of the data acquisition, high dimensionality of data, and high complexity of genomics and proteomics systems. The theme of GENSIPS'11 was cancer and computational biology and GENSIPS featured prominent plenary speakers including Dr. John N. Weinstein from UT MD Anderson Cancer Center, Dr. Stephen Wong from Methodist Hospital at Cornell University, Dr. David Nelson from Baylor College of Medicine and Dr. Chung-I $\mathrm{Wu}$ from the Beijing Institute of Genomics.
\end{abstract}

\section{Articles}

This supplement contains extended versions of selected articles from GENSIPS conference proceedings. Each submitted article to the conference was reviewed by a minimum of two reviewers and the top twenty favorably reviewed papers from sixty selected papers $(33 \%)$ were invited to submit the extended versions for this supplement. The extended journal versions were further reviewed according to rigorous peer-review criteria.

The accepted articles can be broadly categorized into four groups:

\footnotetext{
* Correspondence: ranadip.pal@ttu.edu

'Department of Electrical and Computer Engineering, Texas Tech University, Lubbock, 79409, USA

Full list of author information is available at the end of the article
}

Gene network inference and cancer therapy design

Models of genetic regulatory networks can belong to the class of deterministic or stochastic, discrete or continuous, fine-scale or coarse-scale quantitative models. The availability of experimental data, prior biological knowledge and purpose of modeling frequently guide the selection of the quantitative model to represent the biological network. Sridharan et al. [1] provides an approach for Boolean modeling and analysis of the oxidative stress response pathway based on prior biological knowledge. Oxidative stress has been implicated in a variety of diseases including, but not limited to aging and age-related diseases such as cancer, cardiovascular disease, chronic inflammation, and neurodegenerative disorders. Lin and Khatri [2] considers the aberrant behavior of signaling pathways during cancer as faults in an electronic circuit and presents a Max-SAT based automatic test pattern generation (ATPG) algorithm for cancer therapy. In Haider and Pal [3], a Boolean Network inference algorithm from limited time series data utilizing prior biological knowledge on connectivity is presented. The algorithm is validated on synthetic data and experimental transcriptomic measurements from Human Mammary Epithelial Cell line. As compared to two existing Boolean network inference approaches, the proposed algorithm performs better in terms of robustness and estimation of state transitions. Wu et al. [4] provides a linear regression approach to model transcription regulation following estrogen stimulation in breast cancer cells. The proposed technique is validated using gene expression and ER $\alpha$ Chip-seq data from the MCF-7 cell line. Fine-scale modeling of genetic regulatory networks using stochastic master equation models entails a huge computational complexity and Karim et al. [5] presents a computationally inexpensive way to 
generate the steady state distributions for stochastic master equation models. In Wang et al. [6], a quantitative mathematical model to predict macrophage activation patterns following myocardial infarction is reported. The model was validated on experimental data from adult C57 mice. A single type of genomic data is typically not suitable to understand regulation of cell behavior. Vicente et al. [7] presents an approach to assess the gain in predictive performance by integrating various types of biological information in network inference.

\section{Genomic data analysis}

For analysis of DNA methylation profiles, Zhang et al. [8] presents a non-parametric infinite beta mixture model to cluster DNA methylation expression profiles produced by Illumina Infinium Beadchip. For genome-wide association studies (GWAS), Jia and Zhao [9] applies a dense module search algorithm for locating network modules that are jointly associated with a disease. A restricted search approach is applied for reducing the computational complexity and the strategy is demonstrated on CATIE GWAS dataset for schizophrenia. Taslim et al. [10] proposes a quantitative approach using mixture models to characterize patterns of promoter regions and predict novel and alternative promoters. Jahid and Ruan [11] proposes a method for biomarker discovery by combining microarray gene expression profiles and proteinprotein interaction networks.

\section{Prediction of drug effectiveness}

Targeted cancer therapy is considered to be a cornerstone for personalized medicine. Research problems in this area include understanding and modeling the mechanisms of action of molecularly targeted drugs and design of combination drug therapies. Li et al. [12] presents an integrated experimental and theoretical approach to investigate the mechanism of action and identify pharmacodynamic characteristics of targeted agents based on cell-line platforms. Specifically, tumor cell response is analyzed via the use of fluorescent reporters; dynamics of drug efficacy for different dosages are studied using dynamic modeling; and time-varying parameters are estimated using system identification techniques. Lin et al. [2] proposes to predict the ffectiveness of targeted drugs and guide drug selection by modeling the cancer pathway as a Boolean circuit. Kim and Yoon [13] presents an adaptive reference update (ARU) algorithm to search for the optimal drug combination by comparing the response of the current combination against that of the reference combination and beneficially updating the drug concentrations. Application to real and synthetic examples shows that the ARU algorithm outperforms existing algorithms in terms of effectiveness, efficiency, and robustness.

\section{Analysis workflows}

Advancement in measurement technologies provides vast quantities of experimental data on various components of the regulome. This requires new approaches for the systematic generation and analysis of the experimental data. For large-scale protein profiling, Sun et al. [14] provides an integrative model for Liquid Chromatography coupled Mass Spectrometry, and apply it to the systematic analysis of key factors that impact the number of identified peptides and quantified proteins, protein quantification error, differential expression results, and classification performance. The presence of extraneous variables caused by the sample variability can significantly affect the statistical analysis of high-throughput genetic data. Hsu et al. [15] proposes a novel approach using batch effect correction, a sample selection process, and a semisupervised clustering method for reducing the confounding and suppression effects induced by the extraneous variables. Rodriguez et al. [16] presents an analysis workflow that permits researchers to compare DNA methylation profiles across multiple biological or patient groups. Comparisons can be made at particular regions of a chromosome or across the genome as a whole. This workflow and its suite of features can assist biomedical scientists in conducting methylation profiling projects and facilitate meaningful biological interpretation of their data. Characterizing copy-number variation is a basic method to profile normal and diseased tissue samples, but challenges remain in accurately interpreting the data from a single genome and comparative measurements from groups of sequenced genomes. Janevski et al. [17] studies several variations of copy-number analysis approaches to assess the significance and impact of each methodology choice. Huang et al. [18] proposes C2Maps platform as an online bioinformatics resource providing biologists with directional relationships between drugs and genes/proteins in specific disease contexts based on network mining, literature mining, and drug effect annotations. Doderer et al. [19] analyzes pathway consolidation approaches and provides a user-friendly web-accessible tool that can enable users to extract functional relations of genes across multiple pathway databases. A case study of performing personal genome analysis on a cloud computing environment is presented in Evani et al. [20]. The approach can assist researchers in applying existing cloud computing technologies to analyze enormous amount of data generated by next-generation sequencing technologies.

\section{Acknowledgements}

This supplement will not have been possible without the support of the reviewers and program committee members of IEEE Gensips'2011.

This article has been published as part of BMC Genomics Volume 13 Supplement 6, 2012: Selected articles from the IEEE International Workshop on Genomic Signal Processing and Statistics (GENSIPS) 2011. The full 
contents of the supplement are available online at http://www. biomedcentral.com/bmcgenomics/supplements/13/S6.

\section{Author details}

'Department of Electrical and Computer Engineering, Texas Tech University, Lubbock, 79409, USA. '2Department of Electrical and Computer Engineering, University of Texas at San Antonio, 78249, USA. ${ }^{3}$ Department of Epidemiology \& Biostatistics, University of Texas Health Science Center at San Antonio, 78229, USA.

\section{Authors' contributions}

All authors served as editors for the supplement, with RP serving as the Lead Editor. All authors helped write this editorial.

\section{Competing interests}

The authors declare that they have no competing interests.

Published: 26 October 2012

\section{References}

1. Sridharan S, Layek R, Datta A, Venkatraj J: Boolean modeling and fault diagnosis in oxidative stress response. BMC Genomics 2012, 13(Suppl 6): S4.

2. Lin PC, Khatri S: Application of Max-SAT-based ATPG to optimal cancer therapy design. BMC Genomics 2012, 13(Suppl 6):S5.

3. Haider $S$, Pal R: Boolean network inference from time series data incorporating prior biological knowledge. BMC Genomics 2012, 13(Suppl 6):S9.

4. Wu $\mathrm{H}$, et al: A modulator based regulatory network for ERa signaling pathway. BMC Genomics 2012, 13(Suppl 6):S6.

5. Karim M, Buzzard GT, Umulis DM: Efficient calculation of steady state probability distribution for stochastic biochemical reaction network. BMC Genomics 2012, 13(Suppl 6):S10.

6. Wang $Y$, et al: Mathematical modeling and stability analysis of macrophage activation in left ventricular remodeling post-myocardial infarction. BMC Genomics 2012, 13(Suppl 6):S21.

7. Vicente $F$, et al: Assessing the gain of biological data integration in gene networks inference. BMC Genomics 2012, 13(Suppl 6):S7.

8. Zhang L, Meng J, Liu H, Huang Y: A nonparametric Bayesian approach for clustering bisulfate-based DNA methylation profiles. BMC Genomics 2012, 13(Suppl 6):S20

9. Jia $P$, Zhao Z: Searching combined association signals in schizophrenia genome-wide association studies through an integrative network approach. BMC Genomics 2012, 13(Suppl 6):S15.

10. Taslim C, Huang K, Lin S: Integrative genome-wide chromatin signature analysis using finite mixture models. BMC Genomics 2012, 13(Suppl 6):S3.

11. Jahid M, Ruan J: A Steiner tree-based method for biomarker discovery and classification in breast cancer metastasis. BMC Genomics 2012, 13(Suppl 6):S8.

12. Li $X$, et al: Assessing the efficacy of molecularly targeted agents on cell line-based platforms by using system identification. BMC Genomics 2012, 13(Suppl 6):S11.

13. Kim M, Yoon BJ: Adaptive reference update (ARU) algorithm: a stochastic search algorithm for efficient optimization of multi-drug cocktails. BMC Genomics 2012, 13(Suppl 6):S12.

14. Sun Y, Braga-Neto U, Dougherty E: A systematic model of the LC-MS proteomics pipeline. BMC Genomics 2012, 13(Suppl 6):S2.

15. Hsu FH, et al: Reducing confounding and suppression effects in TCGA data: an integrated analysis of chemotherapy response in ovarian cancer. BMC Genomics 2012, 13(Suppl 6):S13.

16. Rodriguez $B$, et al: Methods for high-throughput MethylCap-Seq data analysis. BMC Genomics 2012, 13(Suppl 6):S14

17. Janevski $A$, et al: Effective normalization for copy number variation detection from whole genome sequencing. BMC Genomics 2012, 13(Suppl 6):S16

18. Huang $\mathrm{H}$, et al: C2Maps: a network pharmacology database with comprehensive disease-gene-drug connectivity relationships. BMC Genomics 2012, 13(Suppl 6):S17.

19. Doderer $M$, et al: Pathway Distiller - multisource biological pathway consolidation. BMC Genomics 2012, 13(Suppl 6):S18.
20. Evani US, et al: Atlas2 Cloud: a framework for personal genome analysis in the cloud. BMC Genomics 2012, 13(Suppl 6):S19.

doi:10.1186/1471-2164-13-S6-S1

Cite this article as: Pal et al:: Selected articles from the IEEE International Workshop on Genomic Signal Processing and Statistics (GENSIPS'2011). BMC Genomics 2012 13(Suppl 6):S1.

\section{Submit your next manuscript to BioMed Central and take full advantage of:}

- Convenient online submission

- Thorough peer review

- No space constraints or color figure charges

- Immediate publication on acceptance

- Inclusion in PubMed, CAS, Scopus and Google Scholar

- Research which is freely available for redistribution 